\title{
Spectropolarimetric determination of circumstellar disc inclinations
}

\author{
KENNETH WOOD \\ Department of Physics and Astronomy, The University, Glasgow, Scotland
}

\section{Thomson scattered spectropolarimetric line profiles}

Inference of the density and velocity structure of rotating/expanding circumstellar discs/winds is of considerable interest in the understanding of stellar mass loss. High resolution line spectropolarimetry creates the possibility of diagnosing such envelope structure much more fully than broad band polarimetry or high resolution spectrometry alone since each element of the scattered spectropolarimetric profile picks out the element of the envelope with the appropriate Doppler shift and provides orientation information on it. This problem has been formulated in detail by Wood, et al (1993) for scattering of a finite width line in a flattened envelope - the spectral shape of the scattered Stokes fluxes being determined by isowavelength-shift contours or surfaces which give the relative wavelength shift of the scattered radiation at different regions in the disc. It was also shown how, in the case of a narrow stellar line scattered in a rotating or expanding flat disc with a simply parametrised density and velocity structure (ignoring the smearing effect of electron thermal motions), it is possible to infer the system inclination and structure model parameters from the resulting spectropolarimetric line profile. This poster presents a method for determining the disc inclination from analysis of the scattered profiles - a parameter which cannot be determined uniquely from spectrometry alone - and thus illustrates the powerful diagnostic potential of high resolution line spectropolarimetry.

\section{Determination of disc inclination}

It was shown by Wood \& Brown (1993) that the inclination of a rotating disc, in which the electron thermal velocity was negligible, could be determined from the ratio of the scattered polarised to total fluxes close to the centre of the scattered line, viz,

$$
\frac{F_{\nu}^{Q}(\alpha \approx 0)}{F_{\nu}^{I}(\alpha \approx 0)}=\frac{-\cos ^{2} i}{1+\sin ^{2} i},
$$


where $\alpha=\left(\lambda-\lambda_{0}\right) / \lambda_{0}$ is the dimensionless wavelength shift from line centre, $\lambda_{0}$. The fact that $i$ can be determined results from the fact that use of a scattered spectral line profile, as opposed to broad-band measurements cf Brown \& McLean (1977), allows different parts of the disc to be picked out. Setting $\alpha \approx 0$ picks out a line in the disc in the plane containing the observer and the disc axis along which the scattering angle is either $\pi / 2+i$ or $\pi / 2-i$, giving different values of the scattered total to polarised fluxes.

For a rotating and expanding disc the $\alpha=0$ contour depends on the disc velocity in a complex way prohibiting a simple expression for determining the disc inclination. The inclusion of random thermal velocities is such that the regions of the disc contributing to the scattered flux at a given wavelength are no longer discrete contours and it would appear that the disc inclination may only be inferred for a rotating cold disc according to Eq. 1. However, by considering the total fluxes under the scattered profiles it is possible to determine the inclination uniquely from,

$$
\frac{F^{Q}}{F^{I}}=\frac{\sin ^{2} i}{2+\sin ^{2} i},
$$

where $F^{I}$ and $F^{Q}$ are the integrated scattered Stokes fluxes with the scattered $U$ flux averaging to zero across the line - as would be expected from the polarimetric cancellation properties of an axisymmetric disc. Since only Doppler wavelength redistribution was considered in this scattering analysis the result of Eq. 2 is an obvious one since it is precisely the ratio of the scattered total to polarised broad-band fluxes for any disc as derived by Brown \& McLean.

So, providing the stellar line is narrow so that, apart from the region within the direct line core, the direct and scattered fluxes can be separated then Eq. 2 provides a means of determining disc inclinations irrespective of their velocity profiles. The beauty of this result is that it holds even when the Doppler redistribution due to scattering off thermal electrons is included.

As it stands the analysis presented in this poster for the determination of stellar inclinations is directly applicable to the scattering of narrow photospheric lines in hot, moving circumstellar discs. Forthcoming advances in high resolution CCD spectropolarimetry and the further development of this theoretical framework for interpreting spectropolarimetric line profiles will yield a novel method for determining the structure of stellar winds.

\section{References}

Brown, J.C., McLean, I.S. : 1977, Astron. Astrophys., 57, 141

Wood, K., Brown, J.C., Fox, G.K. : 1993, Astron. Astrophys., 271, 492

Wood, K., Brown, J.C. : 1993, Astron. Astrophys., in press 